\title{
O Core Curriculum da Unesco como Base para Formação em Bioética
}

\author{
The UNESCO Core Curriculum as a Basis \\ for Training in Bioethics
}

Fabiano Maluf Volnei Garrafa

PALAVRAS-CHAVE

- Bioética;

- Educação Médica;

- Currículo;

- Desenvolvimento de Pessoal.

\section{KEYWORDS}

- Bioethics;

- Medical Education;

- Curriculum;

- Staff Development.

A revolução biotecnológica das últimas décadas teve como resultado o desenvolvimento de um poder quase sem limites sobre a vida humana. Tal contexto exige do profissional uma visão globalizada dos problemas éticos e sociais da era contemporânea, alicerçada em sólidas bases filosóficas e legais. Este contexto torna necessária a promoção de novas competências e habilidades relacionadas à vida profissional. Neste sentido, o ensino da Bioética desponta como uma possibilidade de inovação curricular alternativa ao tradicional modelo prescritivo e normativo. Este artigo relata a experiência da Cátedra Unesco de Bioética da Universidade de Brasília com a utilização do Core Curriculum proposto pela Unesco como instrumento didático-pedagógico adequado ao ensino da Bioética. Entre os dilemas pedagógicos enfrentados pela Bioética como disciplina encontram-se: a construção de seus conteúdos, sua estruturação, as concepções teóricas a serem seguidas e seus objetivos. A contextualização e o aperfeiçoamento da estratégia proposta pelo Core Curriculum podem significar importantes instrumentos facilitadores para docentes que buscam organizar práticas didático-pedagógicas inovadoras em Bioética com o intuito de proporcionar resultados efetivos na formação de seus estudantes.

\begin{abstract}
The biotechnological revolution of recent decades has resulted in the development of an almost unlimited power over human life. This context requires the professional to have a global vision on the ethical and social problems of the contemporary era, based on sound philosophical and legal bases. This leads to the necessity of promoting new skills and expertise related to professional life. The teaching of bioethics has therefore emerged as a possible curriculum innovation; an alternative to the traditional prescriptive and normative model. This article aims to describe the experience of the UNESCO Chair in Bioethics at the University of Brasilia using the Core Curriculum proposed by UNESCO as a teaching-learning tool suitable for teaching bioethics. The pedagogical dilemmas faced by bioethics as a discipline include: the construction of its own content, structure, theoretical concepts to be followed, and its own goals. Contextualizing and improving the strategy proposed by the Core Curriculum could represent the development of important facilitating instruments for teachers seeking to organize innovative educational practices in bioethics in order to provide effective results in student training.
\end{abstract}




\section{INTRODUÇÃO}

O caráter eminentemente técnico conferido à formação dos profissionais das áreas da saúde, fragmentado e desarticulado, tem raízes nas recomendações do Relatório Flexner, do início do século $20^{1}$. No campo específico da ética, tal abordagem apresenta um enfoque exclusivamente deontológico, limitado a um conjunto de normas e códigos trabalhados de forma descontextualizada ${ }^{2}$. Essa proposta tem se mostrado insuficiente para atender às necessidades atuais da formação atitudinal e humanística desses profissionais ${ }^{3}$.

$\mathrm{O}$ crescimento e a ampliação do saber científico-tecnológico na segunda metade do século XX e início do século XXI passaram a requerer novos conhecimentos e habilidades que resultaram no surgimento de preocupações e questionamentos de ordem ética não vislumbrados até então ${ }^{4}$. A revolução biotecnocientífica das últimas décadas levou o conhecimento a limites inimagináveis, que resultaram em profundas transformações nas formas de nascer, viver e morrer da humanidade.

A partir destas significativas mudanças, a realidade passou a exigir dos novos profissionais, além de rigoroso preparo específico no campo técnico-científico, uma visão mais globalizada e aplicada dos problemas morais, éticos e sociais, alicerçada em sólidas bases ético-filosóficas e legais. Para atingir esse objetivo, é necessário promover o desenvolvimento de novas competências e habilidades relacionadas com as questões éticas cotidianas e com a própria práxis exercida por esses profissionais $\mathrm{s}^{5-8}$. Nesse contexto, o ensino da Bioética desponta como uma possibilidade de inovação curricular alternativa ao antigo modelo deontológico prescritivo e normativo baseado em obrigações e deveres morais absolutos.

No entanto, segundo Azevedo", "pelo fato de a Bioética ser uma área de saber complexa e recente, não existe, para seu ensino, uma tradição pedagógica específica nem uma experiência didática consolidada" (p. 135). Assim, um processo de ensino-aprendizagem da Bioética pertinente às novas necessidades se tornou um grande desafio a ser superado na atual fase de ampliação teórico-conceitual e prática não somente da disciplina, como também das próprias ciências biomédicas e da saúde.

Como disciplina acadêmica, a Bioética não se limita ao que-fazer pedagógico tradicional, tampouco está delineada numa fundamentação pragmática baseada em teorias preestabelecidas. Trata-se de uma área multi, inter e transdisciplinar ${ }^{10}$ relacionada tanto com o estudo de velhos conflitos morais que se mantêm na agenda internacional desde a Antiguidade (aborto, eutanásia, exclusão social), como com novos problemas decorrentes do progresso científico e tecnológico (novas tecnologias reprodutivas, transplantes de órgãos e tecidos hu- manos, genômica) que acontecem diuturnamente em diferentes lugares e culturas em todo o mundo.

A forte proximidade histórica da Bioética com a deontologia ou ética profissional nos currículos de estudo das profissões de saúde, aliada à diversidade de temas éticos persistentes e emergentes a serem trabalhados, acabou originando grande variedade de abordagens didáticas, que, apesar de despertarem pouco interesse entre os estudantes, dificultam aos docentes a escolha e organização de conteúdos para o adequado ensino da disciplina.

Com este panorama é que pesquisadores vinculados aos programas educativos de ética da Unesco organizaram um Core Curriculum (CC) para o ensino da Bioética. Esse documento - que tem por base os 15 princípios constantes da Declaração Universal sobre Bioética e Direitos Humanos (DUB$\mathrm{DH})^{11}$ - apresenta elementos teóricos, indicações de material instrucional e casos complexos com questões para discussão que têm por finalidade facilitar a organização da prática docente, melhorando a compreensão dos estudantes acerca de situações conflitivas tratadas no âmbito da Bioética.

Torna-se pertinente, portanto, a busca de novas alternativas às abordagens tradicionais para o ensino da Bioética, mais participativas, dinâmicas e atrativas ao olhar discente. Neste sentido, o presente artigo tem como objetivo relatar a experiência de cinco anos da Cátedra Unesco de Bioética da Universidade de Brasília (UnB), com a utilização do Core Curriculum na disciplina "Introdução à Bioética" como instrumento didático-pedagógico viável para o ensino da matéria na graduação.

\section{O CORE CURRICULUM DA UNESCO: ORIGEM E APLICABILIDADE PRÁTICA}

Para iniciar a análise do assunto, é importante compreender a origem do Core Curriculum de Bioética da Unesco, a qual se deu num contexto histórico importante para o ensino da disciplina. O referencial teórico, como dito acima, foi a DUBDH, aliado a outras iniciativas em educação ética empreendidas pela Unesco.

A inclusão da Bioética na agenda temática da Unesco ocorreu em 1993, ocasião em que foi criado seu Comitê Internacional de Bioética (CIB), com a responsabilidade de estabelecer um arcabouço legal internacional para a proteção do genoma humano. Em 1997, após muitas reuniões e discussões, a Organização adotou a Declaração Universal do Genoma Humano e Direitos Humanos, elaborada pelo referido comitê e homologada em assembleia da qual participaram os países componentes da mesma ${ }^{12}$.

Em 1999, a Conferência Mundial sobre Ciência, por meio da Declaração sobre Ciência e o Uso Científico do Conheci- 
mento, propôs que os princípios éticos e a responsabilidade científica deveriam ser parte integral da educação e da formação de todos os pesquisadores. Em 2002, a educação ética foi considerada pela Unesco uma das cinco principais prioridades para o período compreendido entre 2002 e $2008^{13}$.

Por ocasião da 32ª Conferência Geral da Unesco, em 2003, ficou expresso por muitos países o desejo de estimular iniciativas que promovessem programas de educação ética e não somente de bioética. Nesse sentido, a Comissão Mundial sobre Ética do Conhecimento Científico e Tecnológico (World Commission on the Ethics of Scientific Knowledge and Technology - Comest) publicou em 2003 o relatório O ensino da ética, que passou a estimular o ensino do tema em todos os níveis de educação ${ }^{13}$.

Deste modo, a implantação do Programa de Educação Ética criado em 2004 com o apoio dos 191 estados-membros visou reforçar o ensino da ética em escolas e universidades para todos os pesquisadores e profissionais da educação ${ }^{14,15}$. O conteúdo sugerido não determinava ou impunha nenhuma visão ou modelo ético ou bioético específico; apenas articulava os princípios já compartilhados e referendados por especialistas da área, por gestores e profissionais de saúde. Esta percepção se ateve à necessidade de respeitar as diversidades religiosas, culturais e históricas nos diferentes contextos.

Em outubro de 2005, a 33 a Conferência Geral da Unesco homologou a DUBDH com o voto unânime de seus 191 estados-membros, com o comprometimento de aplicar os princípios e fundamentos da bioética que respeitem a dignidade e a liberdade humana, incluindo as questões e decisões éticas relacionadas à medicina, às ciências da vida e às tecnologias, com o objetivo de proteger e garantir o respeito à vida dos seres humanos ${ }^{16}$.

A DUBDH se ocupa dos princípios básicos da bioética, tais como a dignidade humana e os direitos humanos, a autonomia e a responsabilidade individual, o consentimento, o respeito pela vulnerabilidade humana e integridade pessoal, a confidencialidade, a igualdade, a justiça, a equidade, bem como de temas relacionados com a responsabilidade social e saúde, o preconceito e a responsabilidade para com as gerações futuras, o meio ambiente e a biodiversidade ${ }^{16}$.

A aplicação prática destes princípios pelas nações signatárias deveria se dar na tomada de decisões e no tratamento de questões bioéticas, na implementação de comitês de ética multidisciplinares, na avaliação e gestão de riscos, nas práticas transnacionais. Foi acordado que a promoção da DUBDH se daria de diversas maneiras, dentre as quais a cooperação internacional e a educação, formação e informação em bioética, como se pode observar no Artigo 23, que propõe que para:
[...] alcançar uma melhor compreensão das implicações éticas dos avanços científicos e tecnológicos, em especial para os jovens, os Estados devem envidar esforços para promover a formação e educação em bioética em todos os níveis, bem como estimular programas de disseminação de informação $e$ conhecimento sobre bioética ${ }^{16}$. ( $\left.p .11\right)$

Com a finalidade de cumprir este artigo, um grupo de pesquisadores vinculados à Unesco elaborou, em 2008, o Core Curriculum no intuito de apresentar os artigos da DUBDH a estudantes universitários ${ }^{11}$, sobretudo àqueles residentes nos países em desenvolvimento ${ }^{14}$. O documento indicou a possibilidade de o CC ser útil não somente para a formação pós-acadêmica, mas também para a educação continuada e para programas educacionais dirigidos a membros de comitês de ética ${ }^{11}$.

Esse grupo, ad hoc, era composto por um Comitê Consultivo de Especialistas sobre o Ensino da Ética (Advisory Expert Committee on the Teaching of Ethics) com a presença de representantes da Comissão Internacional de Bioética; da Comest; das Cátedras Unesco de Bioética espalhadas pelo mundo; da Academia de Ciências para o Desenvolvimento Mundial (TWAS); e da Associação Médica Mundial ${ }^{13}$.

A abordagem metodológica do CC proporciona subsídios didáticos essenciais, como orientações gerais aos docentes, indicação de carga horária mínima de estudo e o respectivo conteúdo programático para o adequado ensino de cada tema. É indicado não somente para estudantes das áreas biomédicas e da saúde, mas também para cursos das áreas humanas que tenham interface com a disciplina, como Filosofia, Direito, Antropologia e Sociologia.

A possibilidade de inovação e a flexibilidade de aplicação ao ensino em diferentes contextos são características essenciais do modelo curricular proposto. Deste modo, o CC não constitui uma proposta fechada, podendo, inclusive, ser utilizado como uma fonte complementar às demais abordagens de ensino em Bioética.

A estrutura do programa tem carga horária aproximada de 60 horas e é composta por 17 unidades temáticas. As unidades temáticas 1 e 2 proporcionam uma aproximação geral da ética e da Bioética e são seguidas por mais 15 unidades sequenciais que abordam e discutem os 15 artigos da DUBDH. A proposta da Unesco sugere que algumas das unidades podem ser ministradas conjuntamente, mas cada uma elas desenvolve um tema específico. Pode-se destacar que cada unidade apresenta elementos teóricos fundamentais para a compreensão do tema, bem como exemplos atuais e relevantes que podem ser adequados à diversidade de contextos culturais relacionados com a realidade onde estão sendo apresentados. 
São três os objetivos gerais de aprendizagem propostos pelo CC: capacitar os estudantes para identificar questões éticas das práticas biomédicas; fornecer elementos teóricos para que os estudantes apresentem justificativas racionais para a tomada de decisão ética; capacitar os estudantes na aplicação dos princípios da DUBDH.

De acordo com a Unesco, a utilização do CC em sala de aula, assim como em qualquer curso universitário, deve dispor de estratégias de avaliação. A proposta envolve a avaliação dos estudantes (exames orais, ensaios, análises de casos, provas com questões de múltipla escolha, trabalhos e testes escritos) e do próprio CC. Por meio de um questionário padronizado que acompanha a proposta, estudantes e professores podem avaliar o método utilizado, com o objetivo de dar retorno à Unesco e contribuir para a sua melhoria ${ }^{14}$.

Mais recentemente, o grupo de pesquisadores responsável pela elaboração do CC construiu materiais complementares, como um manual para o instrutor, bem como casebooks para auxiliar a discussão de algumas unidades ${ }^{14,15,17,18}$. O manual para o instrutor detalha cada unidade, reforça objetivos centrais de aprendizagem e orienta quanto aos materiais complementares. Sugere também estratégias para maximizar a participação dos estudantes, como, por exemplo, em que momentos do desenvolvimento da proposta se devem organizar grupos específicos de discussão ou utilizar determinado documentário.

\section{A EXPERIÊNCIA DA CÁTEDRA UNESCO DE BIOÉTICA DA UNIVERSIDADE DE BRASÍLIA}

Considerada como tardia ${ }^{19}$, a bioética brasileira, já em seus primórdios, na década de 1990, buscava uma reflexão autóctone, voltada à dimensão social e direcionada principalmente aos dilemas vivenciados no campo da saúde pública e coletiva ${ }^{20}$. De acordo com Gomes et al. ${ }^{21}$, o desenvolvimento mais orgânico do ensino e da pesquisa em bioética teve início nesse período, mais precisamente em 1994, com a criação do Núcleo de Estudos e Pesquisa em Bioética (NEPeB), vinculado ao Centro de Estudos Avançados Multidisciplinares (Ceam) e à Faculdade de Ciências da Saúde, ambos da Universidade de Brasília (UnB), que desde seu início foi cadastrado formalmente como "grupo de pesquisa consolidado" junto ao Conselho Nacional de Desenvolvimento Científico e Tecnológico (CNPq).

É oportuno registrar as transformações ocorridas no referido Núcleo durante esse período com relação às suas características institucionais e à própria formulação do conhecimento científico no campo da bioética. No nível acadêmico, foi formalmente apresentado à comunidade acadêmica internacional no Sexto Congresso Mundial de Bioética, em 2002, com a inclusão da sua proposta de "Bioética de Intervenção" (BI) como uma das conferências de abertura do evento, que reuniu pesquisadores de 62 países.

Esta proposta epistemológica tem uma fundamentação teórica que de certo modo busca recuperar o ponto de partida da disciplina de acordo com a ideia original proposta por Van Ressenlaer Potter em 1970. Neste contexto, a BI se propôs ampliar o âmbito de atuação da bioética para a dimensão social, ratificando a transdisciplinaridade como estratégia de abordagem bioética e incorporando os direitos humanos como eixo orientador da ética nas práticas coletivas ${ }^{21,22}$.

No nível institucional, destacam-se: o reconhecimento pela Unesco das atividades desenvolvidas pelo NEPeB, conferindo-lhe o título internacional de Cátedra em 2004 e, mais recentemente, em 2008, a criação do Programa de Pós-Graduação Stricto Sensu (mestrado e doutorado) em Bioética, pioneiro nacionalmente nas universidades públicas ${ }^{21}$.

Desde 2010, a Cátedra Unesco de Bioética da UnB adotou o Core Curriculum como instrumento didático para o desenvolvimento da disciplina "Introdução à Bioética", ofertada todos os semestres pela Faculdade de Ciências da Saúde da UnB desde 1994. Durante esse período, embora a disciplina seja direcionada preferencialmente aos estudantes das áreas biomédicas e da saúde, atendeu também a estudantes de outras áreas de conhecimento, especialmente das ciências sociais e humanas.

Assim, seguindo a proposta original da Unesco, foram incorporados os artigos da DUBDH às discussões das situações persistentes e emergentes em bioética, adequando os conteúdos do CC à realidade brasileira. A Cátedra Unesco de Bioética da UnB, então, passou a propor como meta principal contribuir para o aprimoramento de estratégias de ensino da Bioética entre os estudantes, partindo da compreensão de que o Core Curriculum não é "um curso completo, mas fonte de inspiração"11 e de que seu uso deve ser contextualizado às idiossincrasias culturais, morais e sociais de cada cultura, grupo social ou região que o adota.

A disciplina "Introdução à Bioética" tem dois créditos (30 horas/aula) e é desenvolvida em 15 semanas em cada semestre letivo, sendo oferecida a duas turmas de 50 alunos cada. Uma delas é desenvolvida exclusivamente por um professor com especialização, mestrado e doutorado em Bioética, e a outra tem uma peculiaridade que merece ser relatada. É coordenada por professor credenciado no Programa de Pós-Graduação Stricto Sensu da UnB e serve como base para os estudantes de doutorado desenvolverem a disciplina obrigatória "Estágio de Docência em Bioética" (4 créditos - 60 horas/aula).

Com parte do referido "estágio", quatro estudantes de pós-graduação matriculados no semestre, sob orientação e 
com a presença do professor responsável, organizam as aulas e seminários. Cada um deles tem a incumbência de preparar, discutir antecipadamente com os demais colegas matriculados no estágio, apresentar e conduzir pelo menos três destas aulas, que são seguidas por intensos debates com os alunos da graduação que frequentam a disciplina.

Uma semana antes de cada aula, os alunos do estágio têm o compromisso de distribuir, via internet, aos alunos da graduação matriculados na disciplina, um texto de apoio para leitura prévia com o tema a ser discutido, o qual foi produzido pelos estagiários na disciplina do domínio comum do Programa de Pós-Graduação por eles já cursada e denominada "Bases Conceituais da Bioética" (4 créditos). Nesta disciplina, todos estes conteúdos são apresentados e discutidos, gerando os textos de apoio que depois são distribuídos, como descrito acima.

O conteúdo de "Introdução à Bioética", baseado no Core Curriculum da Unesco, tem o seguinte conteúdo sequencial: (1) O que é Ética; o que é Bioética; histórico e bases conceituais da Bioética; a DUBDH da Unesco; (2) Direitos humanos e dignidade humana; (3) Benefício e dano - os princípios da beneficência e não maleficência; (4) Autonomia, consentimento, pessoas sem capacidade de consentir e responsabilidade (individual e pública); (5) Respeito pela vulnerabilidade humana e pela integridade pessoal; (6) Privacidade e confidencialidade; (7) Primeira avaliação teórica; 8) Não discriminação e não estigmatização; (9) Igualdade, justiça e equidade; 10) Respeito pela diversidade cultural e pluralismo; (11) Solidariedade e cooperação; (12) Responsabilidade social e saúde; (13) Solidariedade, cooperação e benefícios compartilhados; (14) Sustentabilidade e proteção das gerações futuras, da biosfera e da biodiversidade; (15) Avaliação final.

Além das duas avaliações teóricas aplicadas na metade e no final do desenvolvimento da disciplina, os alunos de "Introdução à Bioética" são avaliados pela participação ativa nas discussões e na demonstração de leitura prévia e conhecimento dos temas apresentados.

Os artigos da DUBDH incluídos na disciplina "Introdução à Bioética" com base no CC, portanto, situam o conhecimento da área dentro da realidade concreta, abrindo novas perspectivas - mais amplas no sentido biopsicossocial - para reflexão e ações da disciplina. A Declaração da Unesco é um documento "não vinculante", ou seja, não tem poder de lei. Contudo, sua proposta é instrumentalizar seus seguidores para proporcionar, nas decisões a serem tomadas ou nas práticas a serem desenvolvidas, uma orientação teórica e prática para a elaboração de legislações nacionais e regulamentações profissionais ${ }^{23}$
De acordo com Saada ${ }^{24}$, os avanços técnico-científicos que caracterizam o mundo atual têm impacto sobre a conduta humana, individual e coletiva, sobre as relações interpessoais e sobre os valores morais e éticos que regem e regulam a vida social. Neste sentido, o conjunto dos artigos da DUBDH contempla um rol de condições capacitadas a produzir tal impacto, respondendo objetivamente às demandas por soluções éticas para os problemas atualmente vivenciados no contexto internacional.

Ao se associar a bioética, como campo normativo particular na atenção e cuidado da vida e da saúde, com os Direitos Humanos, como campo normativo universal básico de obrigações morais e jurídicas para todas as formas de vida no planeta, identificam-se os valores fundamentais de uma ética universal pautada no respeito à dignidade humana, na luta pela igualdade de direitos, na liberdade, na justiça, fraternidade e paz ${ }^{25}$.

Arduamente construído e duramente conquistado após quase três anos de intensas discussões realizadas pelos cinco continentes, o conteúdo da Declaração de Bioética da Unesco consolida avanços concretos para o estabelecimento de um novo referencial epistemológico e uma nova agenda temática para a bioética do século $21^{26}$. Uma bioética mais humana, mais palpável e mais próxima dos verdadeiros conflitos persistentes que assolam a maioria dos países periféricos do planeta ${ }^{27}$.

\section{CONSIDERAÇÕES FINAIS}

Uma das dificuldades pedagógicas para o desenvolvimento da Bioética como disciplina é a construção de seus conteúdos, como ela deve ser estruturada, como deve ser definida em termos de domínio das concepções teórico-práticas e de seus objetivos. Tais dificuldades podem ser consideradas os principais desafios a ultrapassar, sobretudo porque a maioria das universidades brasileiras ainda não a considera uma disciplina obrigatória e consolidada, nem em seus conteúdos, nem em seus limites teóricos, e muito menos com relação às metodologias utilizadas para sua consecução.

Novas estratégias e tecnologias de ensino devem ser validadas em contextos diferenciados, a fim de aprimorar sua estrutura e melhorar a eficácia. Neste sentido, a utilização pedagógica do Core Curriculum pode ser um instrumento de grande utilidade, influenciando a formação ética e bioética de estudantes em diferentes situações e problemas relacionados com estes indispensáveis campos do conhecimento acadêmico.

O fortalecimento dos espaços para a discussão de temas bioéticos, aliado à construção de sólida formação teórico-crítica de estudantes, deve constituir uma prerrogativa indispensável, principalmente nos países onde existem marcantes 
desigualdades e iniquidades sociais, como no caso do Brasil. A contextualização e o aperfeiçoamento das estratégias propostas pelo Core Curriculum, fundamentado na Declaração Universal sobre Bioética e Direitos Humanos da Unesco, são uma importante alternativa para docentes que buscam organizar suas práticas didático-pedagógicas em Bioética com o propósito de proporcionar melhores resultados à formação de seus estudantes.

Nas palavras do poeta amazonense Thiago de Mello: "Não tenho caminho novo; o que tenho de novo é o jeito de caminhar".

\section{REFERÊNCIAS}

1. Flexner A. Medical education in the United States and Canada: a report to the Carnegie Foundation for the Advancement of Teaching. New York: Carnegie Foundation for the Advancement of Teaching; 1910.

2. Mascarenhas NB, Santa-Rosa DO. Bioética e formação do enfermeiro: uma interface necessária. Texto Contexto Enferm 2010; 19(2):366-71.

3. Siqueira JE. Educação em bioética no curso de medicina. $\mathrm{O}$ Mundo da Saude 2005; 29(3):402-10.

4. Saada A. Introducción. In: Keyeux G, Penchaszadeh V \& Saada A. (coord.). Ética de la investigación en seres humanos y políticas de salud pública. Bogotá: Universidad Nacional de Colombia. Redbioética Unesco, 2006a, p. 23-32.

5. Mascarenhas NB, Santa-Rosa DO. Ensino da bioética na formação do enfermeiro: interface com a bibliografia adotada. Acta Paul Enferm 2010; 23(3):392-8.

6. Pessalacia JDR, Oliveira VC, Rennó HMS, Guimarães EAA. Perspectivas do ensino de bioética na graduação em enfermagem. Rev Bras Enferm 2011; 64(2):393-8.

7. Finkler M, Verdi MIM, Caetano JC, Ramos FRS. Formação profissional ética: um compromisso a partir das diretrizes curriculares? Trab Educ Saude 2011; 8(3): 449-62.

8. Dantas F, Sousa EG. Ensino da deontologia, ética médica e bioética nas escolas médicas brasileiras: uma revisão sistemática. Rev Bras Educ Med 2008; 32(4):507-17.

9. Azevedo EES. Ensino de bioética: um desafio transdisciplinar. Interface 1998; 2(2):127-37.

10. Garrafa V. Multi-inter-transdisciplinaridade, complexidade e totalidade concreta em bioética. In: Garrafa V, Kottow M, Saada A (orgs.). Bases conceituais da Bioética - enfoque latino-americano. São Paulo: Editora Gaia/Unesco; 2006. p.73-85.

11. Organização para a Educação, a Ciência e a Cultura das Nações Unidas. Bioethics Core Curriculum, section 1: syllabus - Ethics Education Programme. Unesco: Paris; 2008.
12. Organização para a Educação, a Ciência e a Cultura das Nações Unidas. Declaração Universal Genoma Humano e Direitos Humanos. Unesco; 1997.

13. Ten Have H. Unesco's Ethics Education Programme. J Med Ethics 2008; 34: 57-9.

14. Organização para a Educação, a Ciência e a Cultura das Nações Unidas. Bioethics Core Curriculum, section 2: study material - Ethics Education Programme. Unesco: Paris; 2008.

15. Organização para a Educação, a Ciência e a Cultura das Nações Unidas. Bioethics Cases Study. Rushap, Unesco: Bangok; 2010. Disponível em: http://www.unescobkk. org/rushap/ethicseducationcorecurriculum/. Acesso em 16 de dezembro de 2014

16. Declaração Universal sobre Bioética e Direitos Humanos. Brasília: Cátedra UNESCO de Bioética; 2005. Disponível em: http://www.sbbioetica.org.br/wp-content/uploads/2011/11/TEXTODADUBDH.pdf. Acessado em 16 de dezembro de 2014.

17. Organização para a Educação, a Ciência e a Cultura das Nações Unidas. Casebook on human dignity and human rigths, Bioethics Core Curriculum Casebook Series, no 1 , Unesco, Paris; 2011.

18. Organização para a Educação, a Ciência e a Cultura das Nações Unidas. Casebook on Benefit and Harm, Bioethics Core Curriculum Casebook Series, nº 2, Unesco, Paris, 2011.

19. Garrafa V. A bioethical radiograph of Brazil. Acta Bioethica. $2000 ; 6(1): 177-81$.

20. Porto D, Garrafa V. A influência da Reforma Sanitária na construção das bioéticas brasileiras. Ciência \& Saúde Coletiva. 2011; 16(Supl.1): 719-29.

21. Gomes ASO, Rodrigues DLN, Sertão VS, Porto D. Ensino em bioética: breve análise da primeira década do Curso de Especialização da Cátedra Unesco de Bioétiva da UnB. Rev Bras Bioética 2009; 5(1-4):82-105.

22. Garrafa V, Porto D. Intervention bioethics: a proposal for peripheral countries in a context of power and injustice. Bioethics 2003; 17(5-6):399-416.

23. Sané P. Aplicación de la declaración universal sobre bioética y derechos humanos. Rev Bras Bioética 2006; 2(4):43742.

24. Saada A. La Declaración Universal sobre Bioética y Derechos Humanos - ampliación democrática para una sociedad más justa. Rev Bras Bioética 2006b; 2(4):413-22.

25. Tealdi JC. Para uma declaración universal de bioética y derechos humanos: uma visión de América Latina. Rev Bras Bioética 2005; 1(1):7-17. 
26. Garrafa V. Apresentação - Um novo conceito de bioética. In: Garrafa V, Kottow M, Saada A (Orgs). São Paulo: Editora Gaia/Unesco; 2006. p. 09-16.

27. Barbosa SN. A participação brasileira na construção da Declaração Universal sobre Bioética e Direitos Humanos da Unesco. Rev Bras Bioética 2006; 2(4):423-36.

\section{CONTRIBUIÇÃO DOS AUTORES}

Fabiano Maluf: participou da elaboração e redação do artigo; Volnei Garrafa: participou da redação e revisão final do artigo.

\section{CONFLITO DE INTERESSES}

Declarou não haver.
ENDEREÇO PARA CORRESPONDÊNCIA

Fabiano Maluf

Campus Universitário Darcy Ribeiro

Faculdade de Ciências da Saúde

Asa Norte - Brasília

CEP: 70904-970 DF

E-mail: bioetica@unb.br 